\title{
Estudo Exploratório sobre Mundos Virtuais e Agentes Conversacionais na Educação a Distância
}

Aliane Loureiro Krassmann - IFFar, PPGIE/UFRGS, alkrassmann@gmail.com Neiva Larisane Kuyven - UNIFTEC, PPGIE/UFRGS, neivakuyven@acad.ftec.com.br Alex Eder da Rocha Mazzuco - IFFar, PPGTER/UFSM, alexmazzuco@gmail.com Liane Margarida Rockenbach Tarouco - PPGIE/UFRGS, liane@penta.ufrgs.br Magda Bercht - PPGIE/UFRGS, bercht@inf.ufrgs.br

Resumo. Este artigo apresenta um estudo exploratório sobre o uso de Mundos Virtuais integrados com Agentes Conversacionais para promover o senso de presença do estudante, buscando a contribuir com o processo de aprendizagem na Educação a Distância. Este esforço se deu pois os Ambientes Virtuais de Aprendizagem tradicionalmente usados são majoritariamente textuais, e há uma carência de acompanhamento individualizado. Obteve-se relatos positivos de estudantes que utilizaram o ambiente, mas salientou-se algumas dificuldades inerentes ao uso de uma nova tecnologia instrucional, apontando para mudanças necessárias na forma como a abordagem deve ser conduzida.

Palavras-chave: educação a distância; mundos virtuais; agentes conversacionais; senso de presença.

\section{Exploratory Study on Virtual Worlds and Conversational Agents in Distance Education}

\begin{abstract}
This paper presents an exploratory study about the use of Virtual Worlds integrated with Conversational Agents to promote the student's sense of presence, in order to contribute with the learning process in Distance Education. This effort was made because the Virtual Learning Environments traditionally used are mostly textual, and there is a lack of individualized monitoring. It was obtained positive reports of students who accessed the environment, but it was highlighted some difficulties inherent in using a new instructional technology, pointing to necessary changes in the way the approach should be conducted.
\end{abstract}

Keywords: distance education; virtual worlds; conversational agents; sense of presence.

\section{Introdução}

A Educação a Distância (EAD) cada vez mais se distingue como uma modalidade capaz de atender com qualidade aos anseios de universalização do ensino, possibilitando flexibilizar a formação de indivíduos em tempo, espaço, ritmos de aprendizagem e itinerários formativos, o que faz dela a modalidade que mais cresce no mundo (Online Learning Consortium, 2017). Em termos nacionais, dados do INEP (Instituto Nacional de Estudos e Pesquisas Educacionais Anísio Teixeira) mostram que a EAD já é responsável por $21 \%$ dos alunos matriculados em Instituições de Ensino Superior (IES) 
privadas no Brasil, um número que cresceu aproximadamente 55\% entre 2011 e 2015 , chegando a um total de 1.265.359 matrículas em 2015 (FGV/FENEP, 2016).

É comum observar em um curso EAD que, muitas vezes por limitações de ordem administrativa e/ou financeira, ou mesmo pelas características de logística inerentes à modalidade, um mesmo professor atende a uma quantidade de alunos, remota e simultaneamente, que excede o razoável para um acompanhamento próximo das atividades de cada aluno, deixando muitos deles "à deriva".

Além disso, conforme salientado por Alencar e Netto (2014), os tutores acabam destinando boa parte do seu tempo de trabalho à verificação de participação dos estudantes no ambiente, como a leitura de posts de fóruns e entrega de tarefas, diminuindo o período dedicado à interação. Em contrapartida, Mezzari et al. (2013) identificaram a existência de uma correlação positiva e significativa entre a intensidade da participação global dos alunos e os resultados que alcançam.

Em adição a este cenário, pesquisas como as de Lorençatto e Carvalho (2011) identificaram que os AVA usualmente utilizados (baseados na web), por si só, são insuficientes ao se desejar que transcendam o propósito de serem simplórios repositórios de atividades, que tipicamente se concentram em fornecer informações, tarefas e arquivos. Percebe-se também a carência da promoção do senso de presença no estudante (sensação de "estar lá", mais próximo do conteúdo), potencializada pela sua distância física do ambiente acadêmico e sua infraestrutura.

As dificuldades mencionadas, ou seja, a falta de acompanhamento individualizado, o "engessamento" dos AVA tradicionais, bem como a falta do estímulo ao senso de presença, são fatores que podem contribuir para uma visão popularmente encontrada de que a EAD é inferior à presencial em termos de aprendizagem.

Diante deste contexto, este estudo investiga a utilização de um suporte midiático, complementar ao AVA tradicional, com potencial de atuação nas dificuldades mencionadas, composto por Mundos Virtuais e Agentes Conversacionais, especialmente quanto à promoção do senso a presença do estudante de EAD. A etapa exploratória apresentada neste artigo buscou verificar a reação dos estudantes quanto ao uso da tecnologia proposta.

\section{Mundos Virtuais e Agentes Conversacionais}

Durante séculos o texto foi considerado o principal formato para o ensino de material científico, e os livros a principal ferramenta. De forma análoga, computadores ainda são usados, com frequência, como livros de alta tecnologia, que apresentam grandes quantidades de informações em formato de texto (Moreno et al., 2001). Nos dias atuais, o estudante da modalidade EAD muitas vezes ainda se depara com um curso predominantemente textual, apesar da vasta inovação tecnológica disponível para uso.

Como exemplo de inovação tecnológica, existem os Mundos Virtuais (MV), que trazem como diferencial a característica da imersão, em um espaço virtual 3D que permite produzir um sentimento de estar lá (Warburton, 2009), um elemento-chave para criar experiências mais reais e ricas. Nas plataformas de MV comumente utilizadas, como Second Life e OpenSimulator, existem os Non Player Characteres (NPC), avatares controlados por script que podem desempenhar diversos papeis, como guias de navegação no ambiente.

Em MV também é possível estabelecer a interconexão com sistemas externos, por meio de requisições Hypertext Transfer Protocol (HTTP), como Agentes 
Conversacionais (ou chatbots), que são softwares que simulam o diálogo por meio de uma interação em linguagem natural. Dessa forma, um NPC pode começar a funcionar como um companheiro virtual, com o qual é possível interagir.

Entre as vantagens dos Agentes Conversacionais, Fonte et al. (2009) destacam a capacidade de resolver dúvidas como um tutor real, podendo manter uma conversa social ou sobre um conteúdo específico, fornecendo uma maneira adicional de obter informações, em consonância com a crescente interação de humanos com interlocutores digitais e o uso de aplicativos de mensagens (Zumstein; Hundertmark, 2017).

Baseando-se nesses pressupostos, essa combinação de tecnologias é proposta nesta pesquisa, realizando a interconexão de Agentes Conversacionais aos Mundos Virtuais, apresentando-se como um suporte midiático complementar ao AVA tradicional. Propõe-se a utilização do NPC no papel de um companheiro virtual, em razão de seu caráter menos invasivo, mantendo a liberdade e autonomia do indivíduo, o que é especialmente importante em se tratando do público-alvo de estudantes na modalidade EAD, usualmente composto por adultos.

\section{Senso de Presença}

Bulu (2012) afirma que o senso de presença pode ser dividido principalmente em três categorias: presença de lugar (sensação de estar lá), presença social (sensação de estar com outros) e co-presença (sensação de estar junto). Considera-se neste estudo a presença de lugar, pois, de acordo com Blascovich et al. (2002, p. 10), o senso de presença em ambientes virtuais "não é importante apenas em termos da presença de outros ou presença social, mas também em termos do self, ou presença pessoal e, em termos do ambiente ou presença ambiental”.

Nesse sentido, o senso de presença é definido por Witmer e Singer (1998, p. 01) como a "experiência subjetiva de estar em um lugar ou ambiente, mesmo quando se está fisicamente situado em outro". Em termos educacionais, os autores sugerem que um aumento no senso de presença pode acarretar em melhorias na aprendizagem. Isto porque, de acordo com Hassell et al. (2009), com o senso de presença os alunos terão ações e comportamento semelhantes aos do ambiente real, e sua concentração será mais focada na atividade. Makransky, Terkildsena e Mayer (2017) corroboram, sugerindo que o senso de presença pode ser canalizado em processamento cognitivo.

Diversos estudos já foram realizados buscando investigar os benefícios da promoção do senso de presença em Mundos Virtuais, tais como Wang, Petrina e Feng (2017), Maratou, Chatzidaki e Xenos (2016) e Chen et al. (2011), porém os resultados para a aprendizagem ainda são inconclusivos. Como diferencial, esta pesquisa investiga um contexto de educação formal, focando em um público de estudantes de cursos regulares ofertados na modalidade EAD.

\section{Desenvolvimento do Suporte Midiático}

O trabalho está inserido no contexto do Projeto AVATAR (ufrgs.br/avatar), financiado pela CAPES, que tem como objetivo difundir o uso da tecnologia na educação. Uma equipe interdisciplinar ligada ao referido projeto apoiou implementação e manutenção do suporte midiático proposto, composta por profissionais das áreas de Informática e Educação.

Fez-se uso basicamente de dois softwares, escolhidos em razão de seu caráter open source, gratuito e de fácil utilização: a plataforma OpenSimulator, para o Mundo 
Virtual, e o Program-O, que utiliza linguagem AIML (Artificial Intelligence Markup Language), para o Agente Conversacional. O grupo de pesquisa envolvido desenvolveu previamente um sistema web para auxiliar na geração da base de conhecimento AIML, denominado FastAIML (Krassmann et al., 2017). A seguir são apresentados detalhes do desenvolvimento do ambiente no Mundo Virtual e do Agente Conversacional.

\subsection{Desenvolvimento do Ambiente de Simulação sobre Matemática Financeira}

Em uma das regiões do Mundo Virtual do Projeto AVATAR, foi criado um ambiente voltado à aprendizagem de Matemática Financeira, consistindo em um prédio que simula uma empresa de contabilidade, chamada C-Company. Portanto, o design da atividade é de uma simulação role-play (dramatização), com o objetivo de proporcionar ao estudante uma visão profissional ao mesmo tempo em que pratica o conhecimento. Os seguintes tópicos abordados na disciplina são contemplados: porcentagem, juros simples, juros compostos, e sistemas de amortização.

A narrativa gira em torno do contexto da empresa fictícia, onde o estudante recebe o papel de estagiário em seu primeiro dia, e deve passar pelos cinco setores que a compõem: Diretoria de Recursos Humanos (RH), Diretoria de Marketing, Diretoria Comercial, Diretoria Administrativa e Presidência.

Nesse cenário, são dispostos diversos NPC, que "povoam" a empresa, e em alguns casos se expressam corporal (simulando digitação no teclado) e textualmente, passando as orientações via mensagens de texto ativadas por sensores de presença. Os personagens participam de um diálogo roteirizado sobre as práticas da empresa. Buscando evitar a dispersão do estudante, flechas no chão são dispostas para auxiliá-lo na locomoção no interior do prédio, indicando os lugares por onde deve seguir.

A atividade possui elementos básicos de gamificação, por meio de desafios em sequência. Assim, em cada sala que chega, o avatar que representa o chefe do setor faz uma breve explicação sobre seu trabalho (Figura 1 "a") e ao final diz para o aluno sentar em uma cadeira para dar início a um questionário, composto de três questões de múltipla escolha, com cinco opções de resposta. Entre os botões há a opção "6-Ajuda" (Figura 1 "b"). Ao clicá-la, a cadeira do estudante gira para a frente de uma tela, e ele é orientado a tocá-la para dar início a um vídeo curto relacionado ao assunto. Para cada acerto é atribuído um ponto, e ao final é dada a pontuação no questionário (0 a 3).
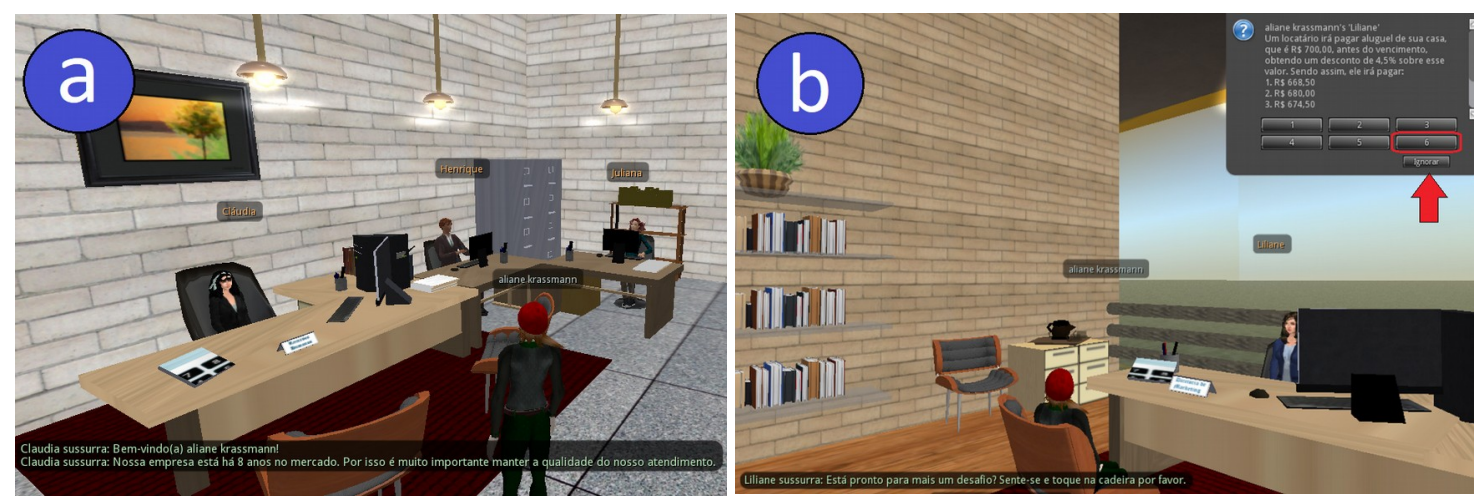

Figura 1. Avatar do estudante em uma das salas do ambiente (a) e questionário (b)

Ao término do questionário, o chefe volta a se comunicar com o estudante, indicando onde está localizada a próxima sala, e assim sucessivamente, em uma concatenação de eventos. A simulação termina quando objetivo de passar pelos cinco 
setores é atingido, levando o aluno a uma sala grande com diversos NPC trabalhando, onde há uma estação de trabalho o esperando para dar início ao seu "estágio".

\subsection{Desenvolvimento do Agente Conversacional}

Neste estudo, pressupõe-se que o acompanhamento de um companheiro virtual pode aumentar o senso de presença e levar a uma percepção mais positiva da experiência. Dessa forma, ao entrar no MV, o NPC Jimmy vem ao encontro do estudante, e se apresenta como um colega na mesma função, passando as instruções e o acompanhando durante toda a interação. Moreno et al. (2001) ressaltam que quando os alunos se identificam com personagens simpáticos, que aparentam estar pessoalmente envolvidos consigo, eles ficam mais propensos a apreciar a situação de aprendizado.

Considerando os estudos de Towns, FitzGerald e Lester (1998), que sugerem reações a serem performadas por agentes, e de Frozza et al. (2011), que desenvolveram um agente companheiro com capacidade de expressar emoções, Jimmy tem capacidade de realizar duas categorias de expressões corporais, a seguir descritas.

- Congratulações: quando o estudante acerta uma questão e quando termina de responder o questionário, Jimmy expressa a emoção de felicidade, com gestos de aplausos ou pulos, apresentando mensagens de congratulações (Figura 2 "a").

- Apoio: quando o estudante erra uma questão, Jimmy se expressa corporalmente abaixando a cabeça ou levando as mãos ao rosto, e verbalmente por meio de mensagens de encorajamento e incentivo à visualização do vídeo de ajuda, também apontando o braço para indicar a disponibilidade de uma calculadora sobre a mesa (Figura 2 "b").
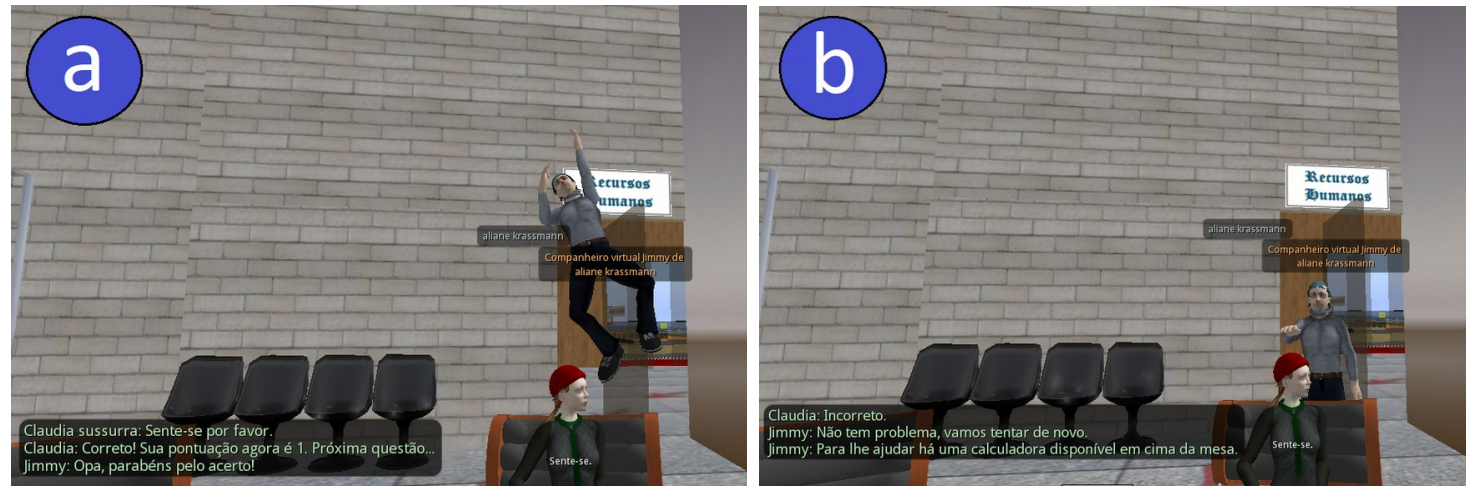

Figura 2. Jimmy após uma resposta correta (a) e apontando para a calculadora (b)

Em concordância com o design do agente de baixa competência de Kim et al. (2006), Jimmy não entra em detalhes sobre como solucionar a questão, mas expressa disposição para trabalhar com o aluno. Dessa forma, serve como um modelo de enfrentamento, buscando aumentar sua confiança e incentivá-lo a continuar a tarefa. A interação em linguagem natural com o Agente Conversacional conectado ao Jimmy ocorre por meio de digitação de mensagens na barra de chat do viewer. Um sensor ativa uma animação no NPC toda vez que uma interação dialógica ocorre, fazendo com que este simule digitar a resposta no teclado.

\section{Procedimentos Metodológicos}

O estudo exploratório faz parte de uma pesquisa ampla de caráter explicativo (causaefeito) e abordagem mista (qualiquantitativa). O design é quase-experimental (não- 
aleatorizado), na medida em que se busca identificar se um tratamento específico influencia um resultado, mas valendo-se de uma amostra de conveniência.

O tipo de suporte midiático é a variável independente, com os sujeitos divididos entre três grupos/condições, sendo: 1) Grupo Real Controle: somente uso do AVA; 2) Grupo Controle: uso do AVA e participação em MV; 3) Grupo Experimental: uso do AVA e participação em MV com companheiro virtual. As variáveis dependentes são o senso de presença e a aprendizagem. Os instrumentos de coleta de dados não serão apresentados em detalhes em razão de não terem sido utilizados nesta etapa.

Propõe-se que o acesso ao Mundo Virtual seja realizado individualmente pelo estudante, para que não haja interferências na mensuração do senso de presença, que é dada pela escala de autorrelato proposta por Witmer e Singer (1998). Além disso, esta participação deve ser de forma voluntária, pois apesar de o serviço estar no modo cliente-servidor, que exime o computador cliente de rodar toda a aplicação, é necessário que este renderize a parte gráfica (3D) dinamicamente. Assim, caso o usuário não detenha de um computador com recursos de hardware mínimos (como placa de vídeo, por exemplo), sua interação pode ser prejudicada ou até mesmo inviabilizada.

Dessa forma, as instruções para download e instalação do visualizador (viewer) Singularity (também gratuito) e suas dependências de software (como Microsoft Visual $\mathrm{C}++$ ), bem como as configurações necessárias para acesso ao MV são disponibilizadas por meio de vídeo tutoriais elaborados pelos pesquisadores.

O estudo exploratório foi conduzido para verificar como seria a reação e aceitação dos estudantes frente ao uso da nova tecnologia, como apresentado a seguir.

\subsection{Estudo Exploratório}

Os pesquisadores entraram em contato com a coordenação do Curso Técnico em Administração, ofertado na modalidade EAD junto ao programa Rede e-Tec Brasil em uma instituição pública do Rio Grande do Sul, apresentando a proposta e convidando-os a testar o ambiente junto à professora de Matemática Financeira. Após o teste, prontamente foi autorizada a condução da pesquisa no contexto do curso, que na ocasião possuía 190 estudantes matriculados (Fevereiro/2018). Porém, em razão da referida disciplina já ter encerrado na ocasião, planejou-se a atividade em caráter espontâneo, sem oferta de gratificações curriculares.

Então, um formulário-convite foi enviado para os e-mails dos alunos, contendo questões sociodemográficas e sobre os recursos computacionais que teriam disponíveis em suas residências. Deixou-se claro que a participação na atividade não influenciaria na nota, mas que o ambiente tinha relação com o curso, mais diretamente com a disciplina Matemática Financeira, sendo uma oportunidade de adquirir e aplicar conhecimentos de forma diferenciada.

Com esta ação, obteve-se a resposta de 64 estudantes. Excluiu-se da listagem aqueles que assinalaram não ter interesse em participar, e os que afirmaram não possuir computador em casa, restando 48 indivíduos. Visando assegurar uma distribuição homogênea de características sociodemográficas, dividiu-se os sujeitos de acordo com os critérios elencados na Tabela 1, considerando-se fatores que poderiam prejudicar na sua disponibilidade de tempo e/ou de recursos para participação, buscando equilibrar o número de indivíduos com mesmas ou similares características em cada grupo.

Tabela 1. Critérios para divisão dos sujeitos da pesquisa em grupos 


\begin{tabular}{cl}
\hline Item & Critérios \\
\hline 1 & Se trabalha em turno integral \\
2 & Número de filhos \\
3 & Velocidade RAM do computador \\
4 & Se faz outro curso \\
5 & Feminino x Masculino \\
6 & Frequência de acesso ao AVA \\
\hline
\end{tabular}

Diante dos três grupos ideais com 16 estudantes cada, criou-se no AVA do curso uma "disciplina", um espaço para passar orientações para cada grupo, sendo reforçado o contato via e-mail. Ofertou-se no espaço destinado ao Grupo Real Controle o mesmo conteúdo (vídeos e questões) a que tinham acesso os demais alunos, incluindo um vídeo com uma visão de como funcionam os setores de uma empresa. Dividiu-se o conteúdo em aulas com duração de uma semana cada, que eram gradualmente disponibilizadas.

\section{Resultados}

Planejou-se o estudo exploratório para ocorrer dentro do período aproximado de um mês. Nesse período, do Grupo Real Controle $(n=16)$ um total de sete estudantes participou de pelo menos uma das atividades propostas no AVA. Dos grupos Controle e Experimental $(\mathrm{n}=36)$, oito estudantes deliberadamente manifestaram não desejar participar mais da pesquisa. Entre os demais, 13 estudantes demonstram algum interesse, respondendo às mensagens, recebendo credenciais de acesso ao $\mathrm{MV}$, e tirando dúvidas sobre a instalação do viewer. Contudo, deixaram de retornar os e-mails.

Em razão dessa baixa participação, o período do estudo se estendeu por três meses, em que se continuou periodicamente buscando contato e pedindo a participação dos estudantes. Um deles relatou que até chegou a instalar o viewer, mas por falta de tempo, por considerar o ambiente "demorado", não teria disponibilidade em participar, pois seria "complicado deixar de estudar para seguir no mundo virtual". Outro teve dificuldade em configurar o servidor do MV no viewer, mas conseguiu superá-la com apoio dos pesquisadores, e até enviou um print da tela com seu acesso, perguntando "e agora, o que eu faço?". Porém, não retornou após dadas as orientações.

Assim, ao final de três meses apenas dois estudantes do Grupo Controle de fato realizaram acesso ao MV e participaram da atividade, como mostra a Tabela 2. Em função da baixa participação dos estudantes, optou-se por não apresentar seus dados demográficos e não realizar a aplicação dos instrumentos de coleta de dados.

Tabela 2. Resumo da participação dos estudantes no estudo exploratório

\begin{tabular}{l|c|c|c}
\hline Grupos & Contagem inicial & Interagiram no AVA & Participaram no MV \\
\hline Grupo Real Controle & 16 & $7(\sim 44 \%)$ & NA \\
Grupo Controle & 16 & $9(\sim 57 \%)$ & $2(\sim 13 \%)$ \\
Grupo Experimental & 16 & $4(25 \%)$ & 0 \\
\hline
\end{tabular}

$N A=$ Não se Aplica

Os dois estudantes que participaram da atividade no MV foram convidados a postar em um fórum no AVA suas percepções, contando um pouco sobre sua experiência. Os relatos ipsis litteris são transcritos a seguir.

\footnotetext{
- Aluno 1 - "Consegui fazer acesso ao ambiente sem nenhum problema. Segui os passos do vídeo de instruções e executei sem nenhuma dificuldade. O Mundo Virtual está bem organizado e fácil de se localizar, onde com as flechas indicando o caminho ficou muito fácil de seguir as etapas.
} 
As atividades dentro do mundo virtual também foram de fácil entendimento pois estava tudo muito bem descrito nas interações com os atores virtuais.

Usei um note normal, sem muita memória RAM ou capacidade de processamento, setei nas configurações gráficas em médio e consegui utilizar o ambiente com muita fluidez."

- Aluno 2 - "Tive alguns problemas para conseguir baixar os programas, mas com a ajuda da profe deu tudo certo. Foi uma experiência bem legal que tive com esse programa."

Observa-se no relato dos estudantes que os tutoriais disponibilizados estavam adequados e foram suficientes para permitir o acesso ao MV a partir de seus computadores pessoais, que não necessitaram deter de recursos computacionais muito avançados. Entretanto, salientou-se que o apoio dos pesquisadores se fez indispensável para responder aos questionamentos e passar orientações quanto à instalação do viewer $\mathrm{e}$ suas dependências de software.

Também destacou-se a agradabilidade da experiência e a facilidade de uso do ambiente. Ou seja, demonstrou-se que a proposta de uso de MV como suporte midiático na EAD é factível de ser implementada, e que pode vir a se tornar um recurso complementar nos cursos, para contribuir com as deficiências quanto ao senso de presença, interatividade, e acompanhamento individualizado.

Porém, em razão do índice insatisfatório de participação dos estudantes, notouse a existência de uma certa falta de interesse na apropriação de novos recursos tecnológicos, o que foi o principal fator inibidor do avanço idealizado pelos pesquisadores. Esperava-se, que, por se tratar de um público online composto por adultos, que acessa à Internet frequentemente para obter sua formação, e que são levados a utilizar uma variedade de f digitais, como sites e blogs, não haveria tantas dificuldades com o uso de Mundos Virtuais.

De certa forma, foi possível observar que os estudantes não levaram a proposta a muito "a sério", pois a maioria ignorou as diversas tentativas de aproximação, e uma boa parte manifestou não ter interesse ou disponibilidade de tempo. Vale destacar que o curso onde o experimento foi aplicado é dividido em três semestres, com uma média de cinco disciplinas sendo ministradas simultaneamente em cada semestre, o que é um fator agravante na disponibilidade de tempo dos estudantes.

Portanto, constatou-se a necessidade de remodelagem da proposta para que se adéque à realidade apresentada. Em conversa com a coordenação do curso, decidiu-se inseri-la como uma atividade extra no contexto de uma disciplina futura do mesmo curso (Administração Financeira). Contudo, de participação voluntária mas oferecendose ao estudante uma gratificação curricular no valor 0,25 na disciplina Prática Profissional Integrada (PPI), no contexto de uma atividade com peso de 2,00.

Em adição, verificou-se a necessidade de comparecimento dos pesquisadores nos Polos EAD, tanto para conduzir a instalação dos viewers quanto para a realização de formação com tutores e estudantes sobre o uso da ferramenta. Acredita-se que essa ação aumentará o número de participantes da pesquisa.

\section{Conclusões}

A Educação a Distância vem crescendo exponencialmente, amparada pela evolução de recursos tecnológicos e a ampliação do acesso à Internet. Entretanto, dificuldades como a falta de acompanhamento individualizado e de interatividade dos AVA tradicionais, 
que também carecem de estímulo ao senso de presença, são fatores que ainda contribuem para uma visão popularmente encontrada de inferioridade à modalidade presencial.

Buscando auxiliar na superação das limitações mencionadas, buscou-se investigar o apoio de um suporte midiático composto pelas tecnologias de Mundos Virtuais e Agentes Conversacionais, atuando como companheiros virtuais.

Apesar de o artigo não apresentar um resultado concreto para o objetivo proposto, levantou-se várias questões reais pertinentes ao domínio da $\mathrm{EAD}$, tais como os requisitos tecnológicos e as dificuldades para implementação de novas ferramentas digitais como Mundos Virtuais, e questões relacionadas ao ser humano e seu comportamento, como interesse e motivação, e voluntariado ou recebimento de recompensa. Em certa medida, revelou-se o fator resistência, e que este não se concentra apenas no lado de professores e gestores de cursos, como poderia se imaginar, mas também se sobressaem nos alunos. Além disso, que não se pode assumir que o aluno da online da EAD esteja pronto para incorporar novas tecnologias no curso.

Não obstante ao resultado restrito, identificou-se indícios acerca da factibilidade de implementação e da satisfação dos estudantes quanto ao uso do referido suporte midiático, que são resultados positivos quanto ao potencial da ferramenta como instrumento de mediação pedagógica. Assim, demonstrou-se como a abordagem proposta pode gradualmente vir a complementar o AVA tradicional, elucidando os desafios práticos que devem ser considerados para a sua efetiva realização.

\section{Referências}

Alencar, M., \& Netto, J. F. (2014). TUtor collaborator using multi-agent system. In International Conference on Collaboration Technologies (pp. 153-159). Springer, Berlin, Heidelberg.

Blascovich, J., Loomis, J., Beall, A. C., Swinth, K. R., Hoyt, C. L., \& Bailenson, J. N. (2002). Immersive virtual environment technology as a methodological tool for social psychology. Psychological Inquiry, 13(2), 103-124.

Bulu, S. T. (2012). Place presence, social presence, co-presence, and satisfaction in virtual worlds. Computers \& Education, 58(1), 154-161.

Chen, J. F., Warden, C. A., Tai, D. W. S., Chen, F. S., \& Chao, C. Y. (2011). Level of abstraction and feelings of presence in virtual space: Business English negotiation in Open Wonderland. Computers \& Education, 57(3), 2126-2134.

FGV/FENEP, Fundação Getúlio Vargas, Federação Nacional das Escolas Particulares. (2016). Números do Ensino Privado 2016, $4^{\mathrm{a}}$ edição.

Fonte, F. A., Carlos, J., Rial, B., \& Nistal, M. L. (2009). Tq-bot: an aiml-based tutor and evaluator bot. Journal of Universal Computer Science, 15(7), 1486-1495.

Frozza, R., da Silva, A. A. K., Schreiber, J. N. C., Lux, B., Molz, K. W., Kipper, L. M., \& Sampaio, L. (2011). Agentes pedagógicos emocionais atuando em um ambiente virtual de aprendizagem. RENOTE, 9(1).

Hassell, M., Goyal, S., Limayem, M., \& Boughzala, I. (2009). Being there: An empirical look at learning outcomes in 3D virtual worlds. AMCIS 2009 Proceedings, 733. 
Kim, Y., Baylor, A. L., \& PALS Group. (2006). Pedagogical agents as learning companions: The role of agent competency and type of interaction. Educational Technology Research and Development, 54(3), 223-243.

Krassmann, A. L., Herpich, F., da Silva, Á. S. P., da Silva, A. R., de Souza Abreu, C., Schmitt, M. A. R., \& Tarouco, L. M. R. FastAIML: uma ferramenta para apoiar a geração de base de conhecimento para chatbots educacionais. RENOTE, 15(2).

Lorençatto, M., \& Carvalho, M. J. S. (2011). A distância transacional e a percepção de estudantes. Revista Novas Tecnologias na Educação (RENOTE), 9(2).

Makransky, G., Terkildsen, T. S., \& Mayer, R. E. (2017). Adding immersive virtual reality to a science lab simulation causes more presence but less learning. Learning and Instruction.

Maratou, V., Chatzidaki, E., \& Xenos, M. (2016). Enhance learning on software project management through a role-play game in a virtual world. Interactive Learning Environments, 24(4), 897-915.

Mezzari, A., Rockenbach Tarouco, L. M., Gorziza Avila, B., Ribas Machado, G., Maria Favero, R. V., \& Bulegon, A. M. (2013). Estratégias para detecção precoce de propensão à evasão. RIED. Revista Iberoamericana de Educación a Distancia, 16(2).

Moreno, R., Mayer, R. E., Spires, H. A., \& Lester, J. C. (2001). The case for social agency in computer-based teaching: Do students learn more deeply when they interact with animated pedagogical agents? Cognition and instruction, 19(2), 177213.

Online Learning Consortium. The Distance Education Enrollment Report 2017. Disponível em: https://onlinelearningconsortium.org/read/digital-learning-compassdistance-education-enrollment-report-2017/. Acesso em: 17 de dezembro de 2018.

Towns, S. G., FitzGerald, P. J., \& Lester, J. C. (1998, August). Visual emotive communication in lifelike pedagogical agents. In International Conference on Intelligent Tutoring Systems (pp. 474-483). Springer, Berlin, Heidelberg.

Warburton, S. (2009). Second Life in higher education: Assessing the potential for and the barriers to deploying virtual worlds in learning and teaching. British journal of educational technology, 40(3), 414-426.

Wang, Y. F., Petrina, S., \& Feng, F. (2017). VILLAGE-Virtual Immersive Language Learning and Gaming Environment: Immersion and presence. British Journal of Educational Technology, 48(2), 431-450.

Witmer, B. G., \& Singer, M. J. (1998). Measuring presence in virtual environments: A presence questionnaire. Presence, 7(3), 225-240.

Zumstein, D.; Hundertmark, S. (2017). CHATBOTS--AN INTERACTIVE TECHNOLOGY FOR PERSONALIZED COMMUNICATION, TRANSACTIONS AND SERVICES. IADIS International Journal on WWW/Internet, 15(1). 